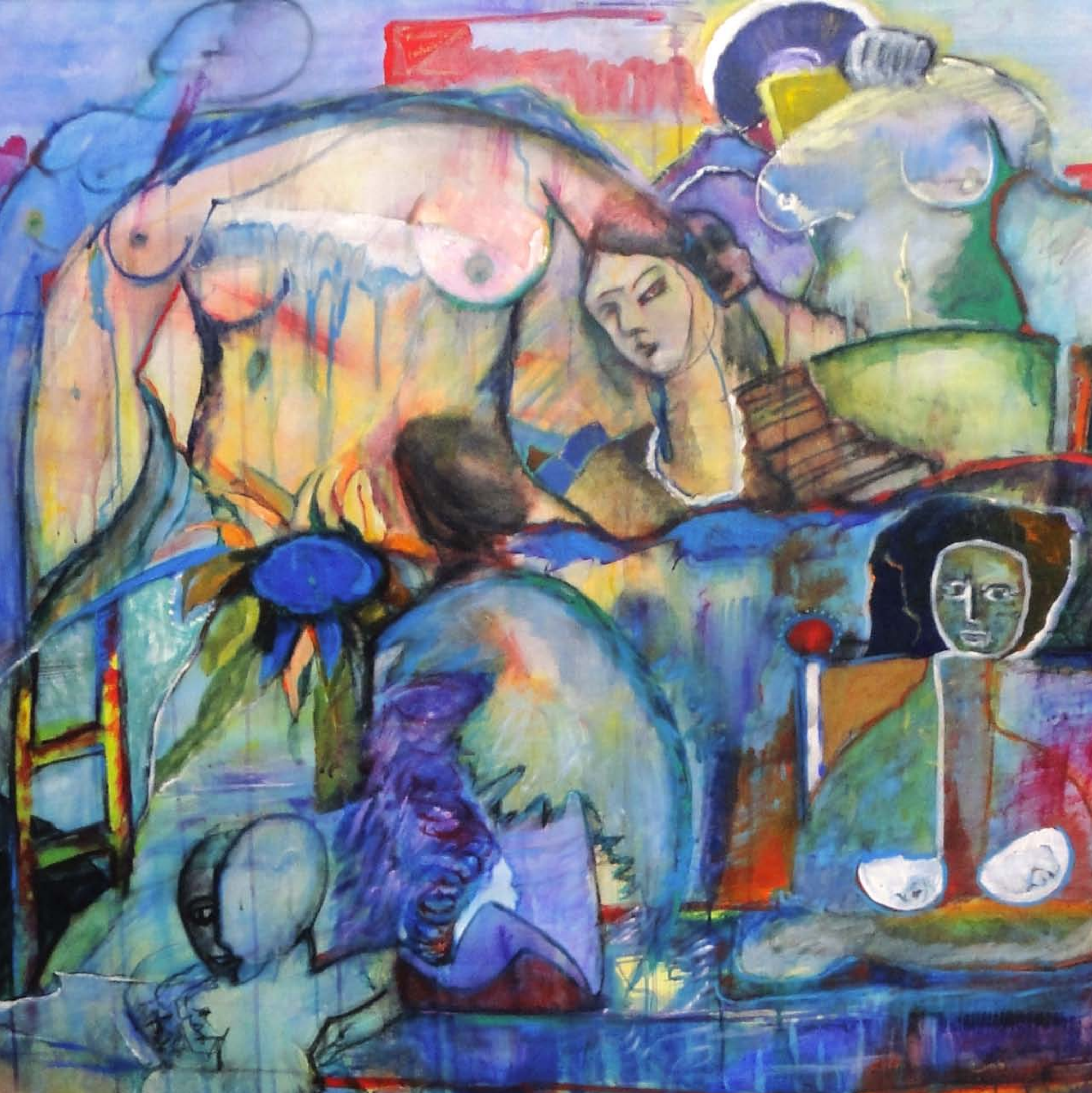




\section{Calidad de la atención a personas sobrevivientes de violencia sexual en el sistema de salud pública en el municipio de La Ceiba}

Adriana Belinda Rodríguez*

María Antonia Nufio**

RESUMEN. La Calidad de la atención a personas sobrevivientes de Violencia Sexual en el sistema de Salud Público municipio de La Ceiba, releva la importancia que tiene el abordaje integral de esta problemática por parte de las instancias involucradas. Siendo la violencia contra las mujeres un problema social y de salud, visibiliza la forma como el sistema desarrolla acciones para la prevención así como para la sanción y el tratamiento cuando se ha producido el hecho. En cuanto a la detección del problema los(as) trabajadores/as de salud, pueden jugar un papel importante, y a su vez, proporcionar, además del tratamiento médico, el apoyo emocional-psicológico, la asistencia social, el acompañamiento y seguimiento a la denuncia. A partir de las capacidades de respuesta del personal de salud ante casos de violencia sexual se revisa las falencias del sistema y se proponen algunas medidas que pueden contribuir a humanizar e implementar un abordaje integral por parte de las instancias de salud y de seguridad.

Palabras clave. Mujeres, víctimas de violencia sexual, sistema de salud público.

SUMMARY. The quality of care to survivors of sexual violence in the Public Health System of the municipality of La Ceiba relieves the importance of a comprehensive approach to this problem by the agencies involved. As violence being a social and health problem against women, visualizes how the system develops actions for prevention as well as to the sanction and treatment when the fact has been occurred. Regarding the detection of the problem, health workers can play an important role, and in turn provide in addition to medical treatment, emotional-psychological support, social assistance, support and monitor of the complaint. From the response capabilities of health personnel, in cases of sexual violence reviews the flaws in the system and proposes some measures that can contribute to humanize and implement a comprehensive approach by the health and security authorities.

Keywords. Women, victims of sexual violence, public health system.

\section{Introducción}

La violencia contra las mujeres es un problema social y un problema de salud por consiguiente meritorio de acciones por parte del Estado para lograr tanto su prevención, como la sanción y tratamiento cuando ya se ha dado el hecho.

Retomando el concepto de la convención de Belém do Pará, la violencia se define como cualquier acción o conducta, basada en su género, que cause muerte, daño, sufrimiento físico, sexual o psicológico, tanto en el ámbito público como en el privado.
Este concepto, reafirma que las mujeres están permanentemente expuestas a diferentes formas de violencia, incluyendo la violencia sexual, que va desde los abusos sexuales de que son objeto en los ámbitos públicos, hasta la violencia sexual que ejercen sus parejas de hogar hacia ellas. Las mujeres que sufren este tipo de agresiones, generalmente asisten a los centros hospitalarios, sin embargo, en muchas ocasiones no hablan del abuso. En este sentido, en la detección del problema los trabajadores/as de salud, pueden jugar un papel importante, y a su vez, proporcionar, además del tratamiento médico, el apoyo emocional-psicológico,

Recibido: enero 2011 / Aprobado y versión final: agosto de 2011.

* Licda. en Psicología UNAH, susimonro@yahoo.com

** Licda. en Trabajo Social, UNAH, antoniant_1986@yahoo.es 
la asistencia social, el acompañamiento y seguimiento a la denuncia.

Siendo la violencia sexual un problema de salud pública, está implícito que toda víctima sobreviviente de violencia sexual (VSVS) debe recibir atención en salud como un derecho fundamental, el cual debe brindarse con eficiencia, eficacia y efectividad; en otras palabras, las VSVS deberían ser tratadas con dignidad. ${ }^{1}$

Entre las distintas manifestaciones de la violencia de género, la violencia sexual es considerada como un grave delito que generalmente se vive en silencio por vergüenza a confesarlo, y por la poca credibilidad en el sistema jurídico del país, lo que vuelve necesario conocer el desempeño de los diferentes actores interrelacionados al sistema de salud pública con los que se comparte responsabilidad legal.

La investigación trata de evidenciar precisamente la calidad de atención que reciben las personas víctimas de violencia sexual por parte de los servidores de salud (médicos, personal de enfermería, trabajadoras sociales y personal de psicología) e identificar las debilidades del proceso de atención a las víctimas de delitos sexuales dentro del sistema de salud en el municipio de La Ceiba.

La investigación incluye un sondeo que expone el conocimiento de la responsabilidad legal así como la toma de conciencia y el compromiso del personal de salud frente a una necesidad vital de protección a la vida y salud integral de las personas sobrevivientes ya que este es un problema considerado como prioritario para el sistema de salud por encontrase dentro de las principales causas de muerte por violencia. Dentro de la responsabilidad del profesional de salud se incluye el establecer una denuncia de los casos asistidos por el Ministerio Público que es la autoridad competente ya que la impunidad es un factor que estimula la frecuencia de los casos de abuso sexual.

\section{Metodología}

Para efectos de la investigación, se realizaron visitas a las seis (6) Unidades de Salud (US) del municipio de
La Ceiba, 2 ubicados en zona urbana, 4 en zona rural y el Hospital Regional Atlántida, realizando entrevistas con personal que da atención directa.

Se consideró aplicar el instrumento de recolección de datos en tres contextos: el Hospital Regional Atlántida, entrevistando al personal médico, de enfermería, psicología y trabajo social, que labora tanto en consulta externa como en la sala de emergencia. En las US, se consideró a personal médico, enfermeras profesionales y auxiliares de enfermería, y el personal de Medicina Forense del Ministerio Público y del Centro Integrado (CEIN).

Otra fuente de información la constituyó la revisión de registros de atenciones hospitalarias, en este caso concreto, el libro de atenciones diarias de la Consejería de Familia; así como el consolidado de atenciones generales en la consulta externa del Hospital Regional Atlántida que recoge específicamente, las 20 causas más comunes de morbilidad para el año 2009.

\section{Contexto del Estudio}

En el municipio de La Ceiba a partir de las dos últimas décadas se ha venido realizando un trabajo de prevención sistemático desde distintas instituciones y organizaciones que trabajan el tema de violencia contra las mujeres. Para el caso desde 1997, nace "La Unidad de Violencia Contra las Mujeres", conformada por las organizaciones e instituciones que trabajan en el tema. Desde esta plataforma, se ha venido haciendo trabajo de incidencia y es así como en junio del 2003 se logra en un cabildo abierto, que la Corporación Municipal apruebe la creación de la Oficina Municipal de la Mujer (OMM).

Otro logro significativo de la red, es la presentación y aprobación de un proyecto de Casa Refugio para mujeres en situación de riesgo por la violencia doméstica y sexual.

De manera incipiente las acciones de prevención, se ven reflejadas en el aumento de las denuncias por parte de las mujeres víctimas de violencia doméstica, quizás no en los niveles deseados pero en comparación a datos de años anteriores si se refleja un aumento. 
3.1 Presencia de instituciones y organizaciones en el trabajo de prevención a la violencia de género.

Actualmente la Unidad de Violencia Contra las Mujeres, continúa en la escena municipal, consolidando procesos preventivos en esta ciudad.

En 1997, se crea en el Hospital Regional Atlántida la Consejería de Familia, cuyo objetivo es la prevención y tratamiento de la violencia intrafamiliar. Los casos de violencia sexual detectados en los diferentes servicios, son referidos a la consejería, la que cuenta con dos personas capacitadas, una psicóloga y una trabajadora social.

Otra instancia importante en la defensa de los derechos de las mujeres, es la Fiscalía de la Mujer. Esta dependencia se creó en el 2008, como una respuesta importante para las mujeres denunciantes de violencia doméstica ya que desde esta Fiscalía se les representa legalmente, así como la investigación y judicialización de casos de femicidios. En el 2009, se inauguró el Juzgado Especial para Casos de Violencia Doméstica.

El Comisionado de los Derechos Humanos, también tiene presencia en esta ciudad y mantiene una estrecha coordinación con las demás instituciones y organizaciones que tienen que ver con derechos humanos.

Es importante mencionar a organizaciones como el Movimiento de Mujeres por la Paz "Visitación Padilla", que desde 1994 implementa acciones en defensa de los derechos de las mujeres, en esta ciudad.

\section{Calidad de la atención brindada por el sector salud del municipio de La Ceiba a sobrevivientes de violencia sexual}

\subsection{Capacidades del personal de salud ante la violencia sexual}

La violencia sexual es un grave problema que afecta a toda la sociedad; es además, un problema de salud pública, que puede ocasionar hasta la muerte de las mujeres, que mayoritariamente son quienes la reciben.

La aseveración anterior, nos lleva a plantearnos entonces la siguiente interrogante ¿Cómo se enfrenta este problema desde la Secretaría de Salud Pública y concre- tamente, desde los centros hospitalarios del municipio de La Ceiba?

En el desarrollo de la investigación se pudo apreciar que el personal capacitado en la temática es escaso y aunque en algunos casos aseguraban haber recibido capacitación, ellos no tienen claridad en los conceptos ni en los procedimientos de atención a víctimas de violencia sexual.

- Personal de salud que atiende víctimas de violencia sexual

Las consecuencias de la violencia sexual, son graves y pueden ir desde un embarazo no deseado, aborto, hasta infecciones de transmisión sexual, VIH/Sida y en algunos casos, intentos de suicidio.

Una atención adecuada y oportuna, es fundamental para mitigar estas consecuencias y contribuir en la erradicación de las causas que la provocan; por ello, en el abordaje a las víctimas de violencia sexual, desde el sector salud, es necesario una atención interdisciplinaria: médica, psicológica y social, con un enfoque de género. Consecuentemente, dicho personal deberá estar previamente capacitado en el tema de violencia sexual y el manejo de estos casos.

Las encuestas realizadas, demuestran que el Hospital Regional Atlántida, no cuenta con un programa de capacitación especializado y que las jornadas de capacitación sobre violencia sexual han sido espontáneas, no sistemáticas, lo que ha impedido muchas veces, que la respuesta institucional, no se dé con un enfoque integral y multidisciplinario.

Es hasta el año 2010, que se inicia un proceso de capacitación sobre el tema, como una iniciativa del Fondo de Población de las Naciones Unidas.

- Formación en violencia sexual

Del personal total entrevistado sólo el 69\% ha recibido orientación en el tema y aún así, algunas personas, no tienen claridad en los conceptos de lo que significa violencia sexual y desconocen los procedimientos a seguir en la atención a un caso de violencia sexual; el 31\% 
restante no ha recibido ninguna formación al respecto. Es lamentable que en este último grupo, el 27\% representado por doctores y doctoras que brindan atención directa a pacientes, denoten poco interés en el manejo a pacientes víctimas de violencia sexual, sobre todo en aquellos casos donde el hecho no es reciente; olvidándose que independientemente del tiempo transcurrido, la violencia sexual siempre es un grave problema de salud y deberá atenderse con calidad y calidez (Ver gráfico No. 1).

\section{Gráfico No. 1. Personal de salud que recibió capacitación} en la temática de violencia sexual

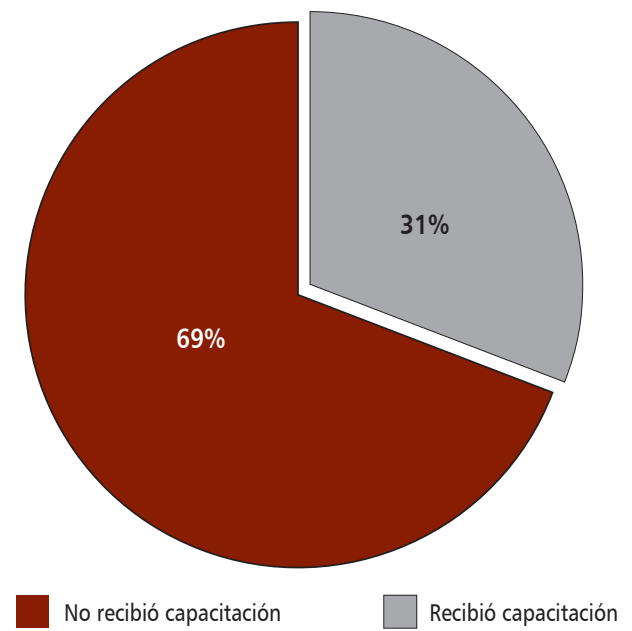

Fuente: Elaboración propia en base a resultados de encuesta aplicada al personal de salud del HLA, 2010.

\subsection{Vulnerabilidad de las mujeres ante la violencia sexual}

Según la Constitución de la República todas las personas en este país tienen iguales derechos y obligaciones indistintamente del sexo; sin embargo, en la cotidianeidad, es evidente que vivimos situaciones desiguales, discriminativas, y de violencia, además son las mujeres las que llevan la peor parte.

Aunque no existen cifras actualizadas sobre la cantidad de mujeres víctimas de violencia sexual atendidas en las unidades de salud, el personal de salud consultado manifestó que de acuerdo a su experiencia la población más afectada por la violencia sexual corresponde a mujeres en edad infantil, tal como lo muestra el Cuadro No. 1 en donde del total de casos registrados, el $37 \%$ se presenta entre los rangos de edad de 5 a 14 años; esto confirma el riesgo y la vulnerabilidad que presentan las niñas ante la violencia sexual, un $26 \%$ se dio en mujeres en un rango de edad de 20-24 años de edad. Un dato importante de resaltar es que el $60 \%$ de los casos reportados corresponde a incestos.

Cuadro No. 1. Hospital Regional Atlántida: casos de violencia sexual, 2009

\begin{tabular}{|l|c|c|c|c|c|c|}
\hline Edad & $5-9$ & $10-14$ & $15-19$ & $20-24$ & $25-39$ & Total \\
\hline No. de casos & 4 & 10 & 9 & 10 & 5 & 38 \\
\hline Porcentajes & 10.5 & 26.3 & 23.7 & 26.3 & 13.2 & 100.0 \\
\hline
\end{tabular}

Fuente: Datos clínica de Consejería Familiar Hospital Regional Atlántida, 2009 una dependencia en el Hospital Regional.

Esta información no pudo ser contrastada con los consolidados de atención del hospital, puesto que se da un sub registro, ya que los/as médicos no están registrando este tipo de diagnóstico, lo que aparece son las consecuencias de la violencia sufrida. Entre los factores que contribuyen a este subregistro se identifican: la falta de conocimiento y sensibilidad ante estas situaciones y el miedo a ser llamados/as a declarar judicialmente por casos concretos ya que el personal teme por "su seguridad" como comentaba una médica.

5.3 Los espacios donde se atiende a las personas víctimas de violencia sexual

Una de las limitaciones que presenta el Hospital Regional Atlántida es la falta de espacios adecuados para atender a las personas víctimas de violencia sexual esto genera limitantes en la atención sobre todo porque las pacientes se encuentran muy vulnerables y sensibles y se requiere generar un ambiente de confianza y privacidad.

Según versión de algunas encargadas de organizaciones que dan acompañamiento a mujeres violentadas, se han presentado manifestaciones de irrespeto por parte de algunos trabajadores(as) de salud: se les mantiene en lugares poco seguros, no siempre se les informa de los 
procedimientos que se les practicarán, el lenguaje tanto verbal como corporal no es el mejor (tono de voz, ser amable, hacer preguntas sencillas y repetirlas si la persona no las entiende).

Otro problema grave es lo referente a la denuncia. La denuncia pronta y efectiva brinda seguridad a la victima y ayuda a combatir la impunidad en que muchas veces queda este tipo de delito.

Según la población entrevistada, no existe el conocimiento de qué hacer en este sentido, unos/as opinan que la denuncia es responsabilidad de la misma persona afectada o sus parientes, otros/as opinan que es el encargado(a) del servicio, consulta externa, emergencia, jefe(a) de los centros de salud quienes deben interponer la denuncia.

Por un lado se identifica la debilidad existente en cuanto al sistema de denuncia y por otro, la falta de respuesta efectiva de parte de los operadores de justicia responsables de este aspecto. Para el caso, la trabajadora social del hospital, se queja de la respuesta tardía del Ministerio Público (agentes de investigación-medicina forense) que permite que en algunas ocasiones, la víctima sea dada de alta sin que lleguen a tomarle la denuncia, se retire del hospital y se pierda el caso.

Una de las sugerencias manifestadas fue la necesidad de procesos de capacitación y guías específicas donde en forma sencilla y clara, se establezcan entre otros datos, los lugares y persona encargada de realizar las denuncias.

\subsection{Una respuesta integral}

La respuesta integral por parte de las instancias intervinientes en la atención de las personas víctimas de violencia sexual debe considerar:

- Atención en crisis, en casos de descompensación.

- Diagnóstico claro.

- Práctica de exámenes oportunos y pertinentes (VDRL, VIH, embarazo).

- Referencia al CAI, para el tratamiento profiláctico de VIH, si no ha transcurrido más de 72 horas del hecho.

- Tratamiento con antirretrovirales si así lo amerita el caso.
- Denuncia del hecho ante las autoridades competentes.

- Referencia a la Consejería de Familia para atención psicológica.

- Seguimiento del caso.

Lamentablemente, no hay respuesta en cuanto a profilaxis por embarazo.

Otra deficiencia encontrada, en la atención a victimas de violencia sexual, es el sistema de referencia y contrarreferencia. La mayoría del personal médico, refiere a la paciente, únicamente cuando el abuso es reciente y se hace necesaria la evaluación por otro especialista o para tratamiento profiláctico de VIH; pero si el abuso se cometió con anterioridad, le restan importancia al apoyo emocional de la persona y no hacen la referencia respectiva.

Algo parecido sucede en el servicio de emergencia, donde la prioridad es la parte física y no se están realizando las referencias a consulta externa para seguimiento.

En la consulta externa funciona la Consejería Familiar donde existe recurso especializado (Psicóloga y Trabajadora Social) que brinda atención en dichos casos, aunque no cuenta con las condiciones adecuadas.

\section{Conclusiones}

- Existe falta de un programa de capacitación sistemático que entregue herramientas teóricas y prácticas para intervenir/atender a personas víctimas de violencia sexual dirigido al personal que trabaja en las Unidades de Salud del municipio de La Ceiba y el Hospital Regional Atlántida; esta carencia impide brindar una respuesta integral a las sobrevivientes de violencia sexual que se presentan a solicitar sus servicios.

- No está protocolizada la atención a personas víctimas de violencia sexual, lo que genera improvisación. Ante la falta de un modelo de atención, la respuesta depende de la sensibilidad o no del o la profesional que atiende en ese momento a la persona demandante del servicio.

- No se cuenta con investigaciones que proporcionen elementos suficientes para la formulación de estrate- 
gias y políticas que prevengan y combatan la violencia sexual.

- La iniciativa propuesta por el UNFPA para desarrollar un programa de capacitación en violencia sexual a personal del Hospital Regional Atlántida, podría ayudar a superar las deficiencias encontradas en el manejo de las víctimas de violencia sexual.

\section{Recomendaciones}

Ante el panorama encontrado, este estudio enfatiza en varias recomendaciones dirigidas a fortalecer los servicios para la atención integral a las víctimas y crear mecanismos eficaces para prevenir y enfrentar la violencia sexual.

- La creación de un modelo de protocolo de atención a víctimas de violencia sexual, que describa con claridad, lo que desde cada servicio, debe hacerse para ofrecer una atención de calidad y calidez a la persona y permita la denuncia oportuna.

- Análisis de modelos ya validados en países latinoamericanos; como el caso concreto del cercano país centroamericano, Guatemala.

- Capacitar al personal de salud especialmente al que brinda atención directa, sobre el manejo de casos de agresión sexual. Esta formación debe orientarse a la atención física, en cuyo caso hablamos de médicos/ as y personal de enfermería; que incluye además de psicólogas/os a trabajadoras sociales debidamente entrenadas.

- Coordinar con el Ministerio Público y Medicina Forense, la capacitación al personal médico sobre evaluaciones físicas en caso de violaciones, toma de muestras y el manejo de posibles evidencias.
- Elaborar e implementar un plan de detección y prevención de la violencia sexual en coordinación con el Ministerio Público y organizaciones que trabajan a favor de derechos de mujer y niñez.

- Fortalecer la Consejería de Familia: reubicarla en un espacio físico que reúna las características requeridas para una atención de calidad. Se le debe dotar de las pruebas psicológicas necesarias para la evaluación de la victima y materiales de apoyo (muñecos sexuados, colchonetas, equipo de música, c d).

- Las y los estudiantes de la carrera de Psicología y Enfermería que realizan su práctica profesional en el hospital, deben ser previamente capacitados sobre la temática de violencia sexual.

\section{Bibliografía}

1. Violencia Sexual en Latinoamérica y El Caribe: Análisis de datos secundarios. Versión: Marzo de 2010.

2. Servicios de salud para Victimas/Sobrevivientes de violencia sexual. IPAS. Octubre del 2009.

3. Documento oficial de las Naciones Unidas. Departamento de Información Pública. (C) Naciones Unidas 2005.

4. Normas, Protocolos y procedimientos para la atención integral de violencia sexual. Bolivia 2005.

5. Protocolo para el abordaje integral de la violencia sexual desde el Sector Salud. Convenio Secretaría Distrital de Salud - Fondo de Población de Naciones Unidas, UNFPA Bogotá, 2008. 


\section{Anexos}

\section{Flujograma de atención a mujeres víctimas de violencia sexual en el Hospital Atlántida}

\section{Flujograma de atención en la consulta externa.}

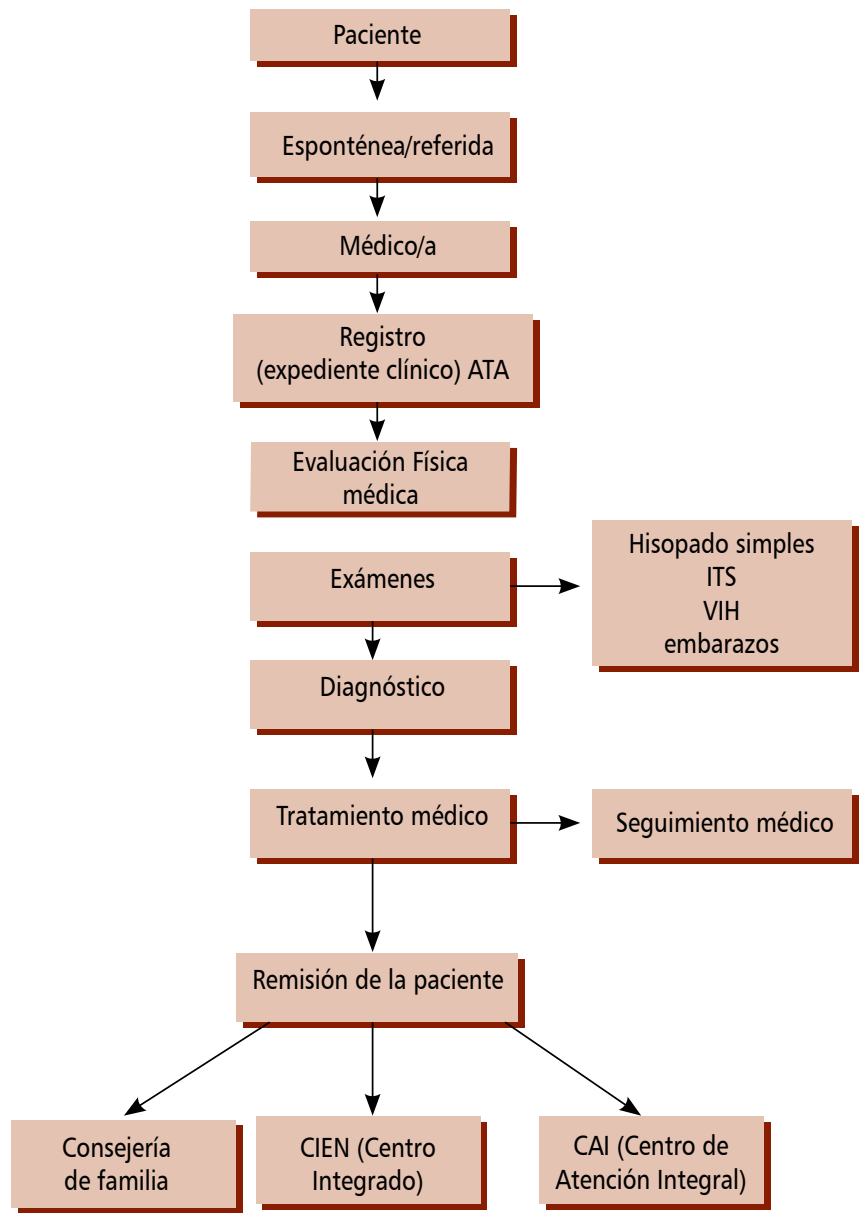

1.1 Descripción de flujograma en el servicio de consulta externa a mujeres víctimas de violencia sexual.

Se atenderá a las mujeres víctimas de abuso sexual:

1. Por demanda espontánea

2. Por referencia de diferentes instituciones y organizaciones como:

- Ministerio Público

- Policía

- Diferentes US
- Emergencia del hospital

- Organizaciones de mujeres

- Organizaciones de derechos humanos

\section{Registro del paciente}

Se registra en el expediente clínico ATA

\section{Evaluación médica}

Esta valoración se hace cuando el abuso ha ocurrido recientemente (días, horas) el cual se complementa a través de la prueba laboratoriales.

Esta valoración se hará sin desestimar la necesidad e importancia del examen de medicina forense

\section{Algunas pruebas son las siguientes}

- VDRL

- VIH

- Hisopados simples

- Prueba de embarazo si es necesario el general de orina y vaginoscopia

- Si el abuso a ocurrido en términos no mayor de 72 horas se ofrecerá tratamiento profiláctico de VIH.

Para el tratamiento profiláctico por VIH el médico/a deberá referirlo al Centro Atención Integral (CAI).

Elaboración de diagnóstico por medio de las pruebas de laboratorio y de un examen físico minucioso.

- Llenar la ficha de violencia sexual y mandar una copia a la dirección del hospital

- En base al diagnostico se decidirá el tratamiento adecuado para cada una de las necesidades encontradas. (ITS, Embarazos, VIH; y otros)

Las referencias o remisiones se harán a:

- Consejería de familia; para el apoyo emocional / tratamiento psicológico, y para el acompañamiento y seguimiento legal.

- CEIN; para interponer la denuncia, si aun no tienen conocimiento del caso. 
- CAI; para el tratamiento profiláctico de VIH y/o tratamiento con retro virales si fuese necesario.

- A otros profesionales que amerite de acuerdo a las necesidades del paciente (médicos especialista, psiquiatra, etc.).

\section{Flujograma de atención en el servicio de emergencia}

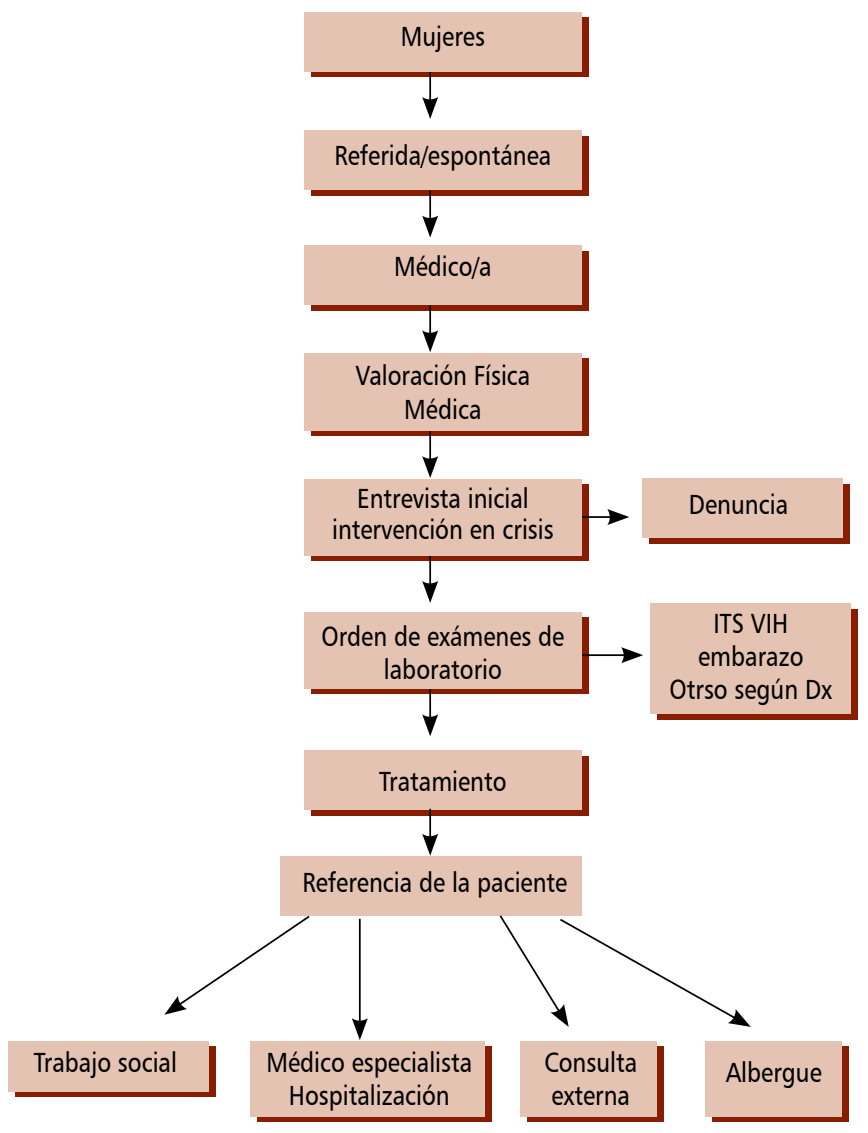

2.1 Descripción de flujograma en el servicio de emergencia a mujeres víctimas de violencia sexual

Se atenderá a las mujeres victimas de abuso sexual:

1. Por demanda espontánea

2. Por referencia de diferentes instituciones y organizaciones como:

- Ministerio Público

- Policía

- Diferentes US

- Organizaciones de mujeres
- Organizaciones de derechos humanos

- Bomberos

- Comunidad

\section{Registro del paciente}

Se registra en el expediente clínico ATA

\section{Evaluación médica}

Esta valoración se hará sin desestimar la necesidad e importancia del examen de medicina forense.

\section{Algunas pruebas son las siguientes}

- VDRL

- VIH

- Hisopados simples

- Prueba de embarazo Si es necesario el general de orina y vaginoscopia.

- Si el abuso a ocurrido en términos no mayor de 72 horas se ofrecerá tratamiento profiláctico de VIH.

Para el tratamiento profiláctico por VIH el médico/a deberá realizar el ínter consulta con personal del CAI.

Elaboración de diagnóstico por medio de las pruebas de laboratorio y de un examen físico minucioso.

La entrevista se realizará con respeto, con calidez y se tratará de recabar la mayor información posible sobre el evento. Si la mujer entra en crisis emocional, se solicitará ayuda a Trabajo Social, quien coordinara con personal de Consejería de Familia, en casos necesarios.

Denuncia

El jefe/ a del servicio, deberá llamar al Ministerio Público para interponer la denuncia, en su defecto lo realizará la jefa de enfermería o el médico/a que atienda la persona.

De igual manera, se informará a la dirección del Hospital

Las referencias o remisiones se harán a:

- Médicos/as especialistas según la situación de la paciente y en caso necesario, se agilizará su hospitalización. 
- Trabajo Social; para el seguimiento del caso

- Consulta Externa: para continuar con tratamiento médico.

- Consejería de Familia; para el apoyo emocional / tratamiento psicológico, y para el acompañamiento y seguimiento legal.

- Albergue: En caso que la mujer se encuentre en grave situación de riesgo, se le refiere al albergue para mujeres víctimas de violencia.

\section{Flujograma de atención en la Consejería de Fami-} lia a mujeres víctimas de violencia sexual

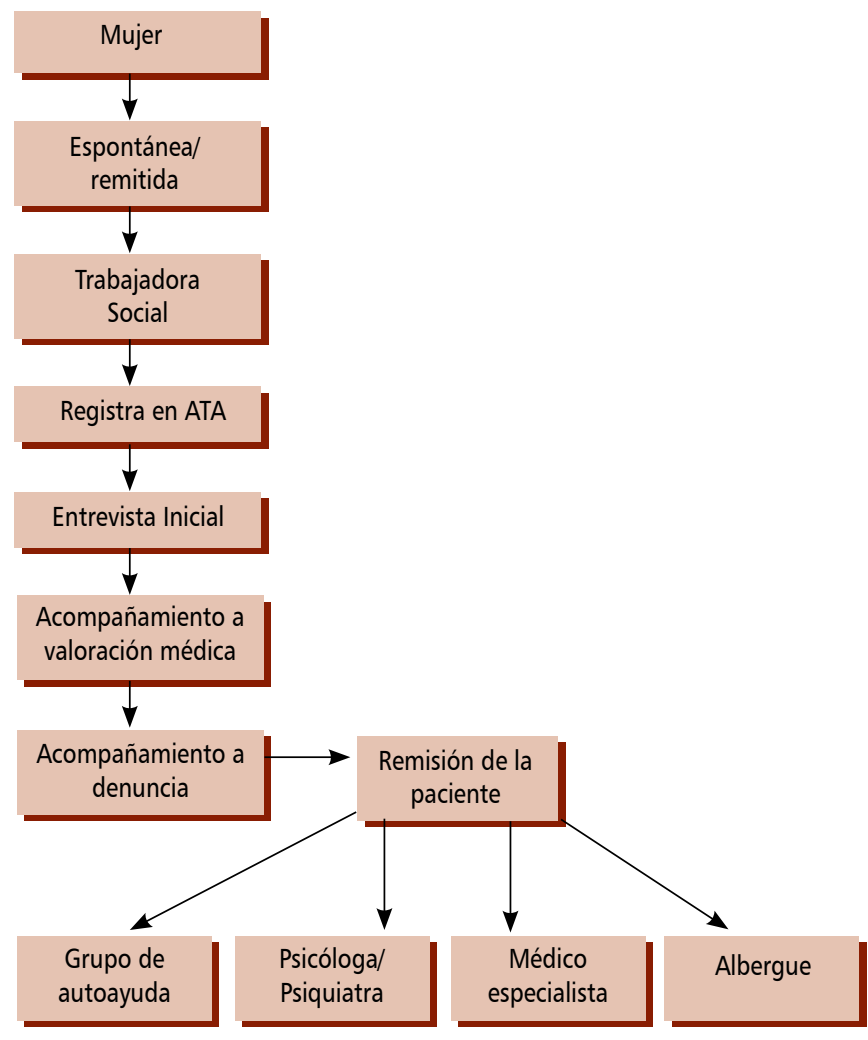

3.1 Descripción de flujograma de atención en la Consejería de Familia a mujeres víctimas de abuso sexual

Se atenderá a las mujeres víctimas de abuso sexual:

1. Por demanda espontánea

2. Por referencia de diferentes instituciones y organizaciones como:

- Ministerio Público

- Policía
- Diferentes US

- Organizaciones de mujeres

- Organizaciones de derechos humanos

- Consulta externa

- Juzgados

- Comunidad

\section{Registro del paciente}

Se registra en el expediente clínico ATA

\section{Entrevista inicial}

Es realizada por la trabajadora social y comprende toda la información socio económica; así como características del evento. Se recoge en un instrumento ya diseñado

\section{Acompañamiento a valoración médica}

En casos que la mujer no quiera interponer la denuncia, se le realizará evaluación ginecológica, para registrar la evidencia del abuso, en el expediente médico.

Se le acompañara así mismo, al CAI, en casos de violación con menos de 72 horas, para exámenes de ITS, VIH y el tratamiento profiláctico por el VIH.

\section{Acompañamiento a denuncia}

Cuando la mujer acepta interponer la denuncia, se le dará acompañamiento si el caso lo amerita.

\section{Referencias}

Se refiere a la psicóloga para tratamiento individual o mediante un proceso de terapia grupal; si presenta un trastorno psiquiátrico, se remite al psiquiatra.

Si se considera en situación de alto riesgo, se refiere al albergue. 